\title{
NONSTANDARD THEORY OF ZARISKI RINGS ${ }^{1}$
}

\author{
LOREN C. LARSON
}

Abstract. Let * $R$ be an enlargement (in the sense of A. Robinson) of a Zariski ring $(R, A)$, let $\mu$ be the monad of zero in ${ }^{*} R$ when $R$ is given the $A$-adic topology and set $R_{\mu}$ equal to the quotient ring ${ }^{*} R / \mu$. It is shown that $\left(R, R_{\mu}\right)$ is a flat couple, and $R_{\mu}$ is Noetherian if and only if it is semilocal. Furthermore, if $R$ is semilocal and $A$ is the (Jacobson) radical then $R_{\mu}$ is semilocal, with the same number of maximal ideals and the same (Krull) dimension as $R$.

1. Introduction. Given a ring $R$ with identity, an $R$-module $M$, and an ideal $A$ of $R$ such that $\bigcap_{n=0}^{\infty} A^{n}=(0)$ and $\bigcap_{n=0}^{\infty} A^{n} M=(0)$, we may make $R$ (resp. $M$ ) into a topological ring ( $R$-module) by adopting $\left\{A^{n} \mid n=1,2, \cdots\right\}$ (resp. $\left\{A^{n} M \mid n=1,2, \cdots\right\}$ ) as a fundamental system of neighborhoods of zero. This topology is referred to as the $A$-adic topology; it is a metric topology and we shall denote the completions of $R$ and $M$ with respect to its $A$-adic topology by $R^{*}$ and $M^{*}$ respectively. If $R$ is Noetherian and each of its ideals is closed in its $A$-adic topology, then $R$ is called a Zariski ring with respect to $A$ and we shall write $(R, A)$ is a Zariski ring. If $R$ is Noetherian, then $(R, A)$ is a Zariski ring if and only if $A$ is contained in its (Jacobson) radical. Noetherian rings having only a finite number of maximal ideals are called semilocal rings. We shall write $(R$; $\left.P_{1}, \cdots, P_{k}\right)$ is a semilocal ring when $R$ is a semilocal ring and $P_{1}, \cdots, P_{k}$ are its maximal ideals.

In this paper we investigate an extension $R_{\mu}$ of $R$ whose construction is familiar to those acquainted with nonstandard analysis and its application to topology and algebra (e.g. [3], [4], [6], [7], [8], [9]). More specifically, $R_{\mu}=* R / \mu$ where $\mu$ is the monad of zero when $R$ is given the $A$-adic topology. Both $R$ and $R^{*}$ are contained in $R_{\mu}$, so as a guide to this study we look for analogues to theorems relating $R$ to $R^{*}$. Two of the deepest of these are that if $(R, A)$ is a Zariski

Presented to the Society, August 29, 1969 under the title Ultrapowers of Zariski rings; received by the editors June $23,1970$.

AMS 1970 subject classifications. Primary 13L05, 13B99; Secondary 13E05, 13 H99.

$K e y$ words and phrases. Zariski rings, semilocal rings, $A$-adic rings, ring completions, ring extensions, flat couples of rings, nonstandard models, enlargements, ultraproducts.

1 This research is an extension of a portion of a doctoral dissertation written at the University of Kansas under the supervision of Paul J. McCarthy and was supported in part by the Research Corporation.

Copyright (c) 1971. American Mathematical Society 
ring, then $\left(R^{*}, A R^{*}\right)$ is a Zariski ring and $\left(R, R^{*}\right)$ is a flat couple. (This definition is due to J. P. Serre $[2$, p. 84]; we mean here that $R$ and $R^{*}$ are rings with a common identity, $R^{*}$ contains $R$ and is a flat $R$-module, and $B R^{*} \cap R=B$ for every ideal $B$ of $R$.) These results are sufficient to prove that the (Krull) dimensions of $R$ and $R^{*}$ are equal and a number of "lying over" type relationships between the ideal structures of $R$ and $R^{*}$. In the next section, after presenting necessary preliminary material, we prove that if $(R, A)$ is a Zariski ring, then $\left(R, R_{\mu}\right)$ is a flat couple. In general $R_{\mu}$ is non-Noetherian and for this reason $\left(R_{\mu}, A R_{\mu}\right)$ may fail to be a Zariski ring. So in the last section we look for conditions on $R$ and $A$ which will make $R_{\mu}$ Noetherian. The main result of this section is that if $A$ is the radical of $R$, then $R_{\mu}$ is Noetherian if and only if $R$ is semilocal.

All rings considered are commutative rings with identity and all modules are unital. The set of natural numbers will be denoted by $I$.

2. Preliminaries; a flat couple. We will assume throughout this section that $(R, A)$ is a Zariski ring and $M$ is a finitely generated $R$ module. Recall then that $\bigcap_{n \in I} A^{n}=(0)$ and $\bigcap_{n \in I} A^{n} M=(0)[12, \mathrm{p}$. 262] and $M$ is Noetherian [11, p. 158].

Suppose $\mathcal{U}$ is a higher order structure which includes $I, R, M$ and any other $R$-modules relevant to the discussion. Let $* \mathfrak{u}$ denote an arbitrary but fixed enlargement of $\mathcal{u}$. (For the definition of enlargement see $\left[7\right.$, p. 819] or $\left[3\right.$, p. 14].) ${ }^{*} M$ is a ${ }^{*} R$-module. If $\mu$ and $\nu$ denote the intersection of all ${ }^{*}\left(A^{n}\right)$ for all finite $n$ and the union of all $*\left(A^{n}\right)$ for all infinite $n$ respectively, then $\mu=\nu[8, \mathrm{p} .447] ; \mu$ is an ideal of ${ }^{*} R$ and we set $R_{\mu}$ equal to the quotient ring $* R / \mu$. We will denote the natural homomorphism from ${ }^{*} R$ to $R_{\mu}$ by $\mu$. (Note that $\mu$ as an ideal and as a homomorphism depends on $A$ for its definition, but since all monads in this paper are computed from the $A$-adic topology on $R$, we will not include this in the notation.) $R$ and $R^{*}$ are contained in $R_{\mu}$ [8, p. 447]; furthermore $R_{\mu}=R^{*}$ if and only if $R / A^{n}$ is finite for each $n$. (Sufficiency is proved in [8, p. 448]. If $R / A^{n}$ is infinite, it can be checked that $\mu\left(a_{w}\right) \notin R^{*}$, where $\left\{a_{i}\right\}_{i \in I}$ is a sequence of representatives from distinct cosets of $A^{n}$ in $R$, and $w$ is an infinite natural number.)

In a manner similar to that in the preceding paragraph, the intersection of all ${ }^{*}\left(A^{n} M\right)$ for finite $n$ is a submodule of ${ }^{*} M$ equal to the union of all ${ }^{*}\left(A^{n} M\right)$ for infinite $n$. Even at the risk of confusion we shall let $\mu$ (resp. $\nu$ ) denote this submodule, and set $M_{\mu}$ equal to the quotient module ${ }^{*} M / \mu$. We will also denote the natural homomorphism from ${ }^{*} M$ to $M_{\mu}$ by $\mu$. The context should make it clear which $\mu$ is being used. 
A very useful result which is applied several times in the paper is that if $Q$ is an internal submodule of ${ }^{*} M$, then $\bigcap_{n \in I}\left(Q+^{*}\left(A^{n} M\right)\right)$ $=Q+\mu$. Half of this equality is trivial. For the other half, let $x \in Q$ $+^{*}\left(A^{n} M\right)$ for all finite $n$. Then $x \in Q+^{*}\left(A^{w} M\right)$ for some infinite natural number $w$. But $Q+{ }^{*}\left(A^{w} M\right) \subseteq Q+\nu=Q+\mu$.

It is easy to check that $\mu(a) \mu(x)=\mu(a x)$ for $a \in^{*} R, x \in \in^{*} M$, is a well-defined product that makes $M_{\mu}$ into an $R_{\mu}$-module. If $N$ is an $R$-submodule of $M$ then $\mu\left({ }^{*} N\right)=\left({ }^{*} N+\mu\right) / \mu$ is an $R_{\mu}$-submodule which we will denote by $N_{\mu}$. Be careful to note that in this context, $N_{\mu}$ does not mean $* N / \mu^{\prime}$ where $\mu^{\prime}$ is the intersection of all finite powers of ${ }^{*}\left(A^{n} N\right)$. However, $\left({ }^{*} N / \mu^{\prime}=\right) N_{\mu^{\prime}} \cong N_{\mu}\left(=\left({ }^{*} N+\mu\right) / \mu\right)$. To see this it suffices to prove that ${ }^{*} N \cap \mu=\mu^{\prime}$ since $\left({ }^{*} N+\mu\right) / \mu \cong * N /\left({ }^{*} N \cap \mu\right)$; that is, we must show that

$$
{ }^{*} N \cap \bigcap_{n \in I}{ }^{*}\left(A^{n} M\right)=\bigcap_{n \in I}^{*}\left(N \cap\left(A^{n} M\right)\right)=\bigcap_{n \in I}^{*}\left(A^{n} N\right) .
$$

But this latter equality follows as an application of the Artin-Rees Lemma [5, p. 9]. Similarly, if $B$ is an ideal of $R$ we set $B_{\mu}=\mu\left({ }^{*} B\right)$.

We note that if $N$ is a submodule of $M$ then $N_{\mu}=R_{\mu} N$; for suppose that $x_{1}, \cdots, x_{k}$ generate $N$. If $\mu(x) \in N_{\mu}$ then $x=y+z$ where $y \in{ }^{*} N, z \in \mu$ and $y=r_{1} x_{1}+\cdots+r_{k} x_{k}$ for some $r_{i} \in{ }^{*} R$. Thus $\mu(x)$ $=\mu\left(r_{1}\right) x_{1}+\cdots+\mu\left(r_{k}\right) x_{k}$ where we have identified $\mu\left(x_{i}\right)$ and $x_{i}$ as usual. That is, $x_{1}, \cdots, x_{k}$ also generate $N_{\mu}$ as an $R_{\mu}$-submodule. An argument similar to that just given shows that if $B$ is an ideal of $R$ and $N$ a submodule of $M$ then $B N_{\mu}=B_{\mu} N_{\mu}=(B N)_{\mu}$. Thus $A^{n} M_{\mu}=\left(A^{n}\right)_{\mu} M_{\mu}=\left(A_{\mu}\right)^{n} M_{\mu}=\left(A^{*}\right)^{n} M_{\mu}=\left(A^{n} M\right)_{\mu}$, so that $A$-adic, the $A^{*}$-adic, the $A_{\mu}$-adic topologies on $M_{\mu}$ coincide, and each agrees with the $S$-topology induced on $M_{\mu}$ by the topology on $M[6, \mathrm{p} .108]$.

Now suppose that $M^{\prime}$ is another $R$-module such that $\bigcap_{n \in I} A^{n} M^{\prime}=(0)$ and that $f: M \rightarrow M^{\prime}$ is an $R$-homomorphism. Define $f_{\mu}: M_{\mu} \rightarrow M_{\mu^{\prime}}^{\prime}$ $\left(M_{\mu^{\prime}}^{\prime}={ }^{*}\left(M^{\prime}\right) / \mu^{\prime}, \mu^{\prime}=\bigcap_{n \in I}{ }^{*}\left(A^{n} M^{\prime}\right)\right)$ by $f_{\mu}(\mu(x))=\mu^{\prime}\left({ }^{*} f(x)\right)$ for $x \in{ }^{*} M$. It is straightforward to check that $f_{\mu}$ is a well-defined $R_{\mu}$-homomorphism.

Lemma. If $M, M^{\prime}$, and $M^{\prime \prime}$ are finitely generated $R$-modules and $M \stackrel{f}{\rightarrow} M^{\prime} \stackrel{\leftrightarrow}{\rightarrow} M^{\prime \prime}$ is an exact sequence of $R$-homomorphisms then $M_{\mu} \stackrel{\rho_{\mu}}{\rightarrow} M_{\mu^{\prime}}$ $\stackrel{o_{\mu}}{\rightarrow} M_{\mu}^{\prime \prime \prime}$ is an exact sequence of $R_{\mu}$-homomorphisms.

Proof. Suppose $\mu^{\prime}(x) \in \operatorname{Ker} g_{\mu}$. Then $\mu^{\prime \prime}\left({ }^{*} g(x)\right)=0$ so that ${ }^{*} g(x) \in \mu^{\prime \prime}=\bigcap_{n \in I} *\left(A^{n} M^{\prime \prime}\right)$. Let $n \in I$ be arbitrary but fixed. $g\left(M^{\prime}\right)$ is a submodule of $M^{\prime \prime}$, hence by the Artin-Rees Lemma, there exists $m \in I$ such that $A^{m} M^{\prime \prime} \cap g\left(M^{\prime}\right) \subseteq A^{n} g\left(M^{\prime}\right)=g\left(A^{n} M^{\prime}\right)$ and therefore

$$
{ }^{*}\left(A^{m} M^{\prime \prime}\right) \cap{ }^{*} g\left({ }^{*} M^{\prime}\right) \subseteq{ }^{*}\left(g\left(A^{n} M^{\prime}\right)\right)={ }^{*} g\left({ }^{*}\left(A^{n} M^{\prime}\right)\right) .
$$


It follows that ${ }^{*} g(x) \in^{*} g\left({ }^{*}\left(A^{n} M^{\prime}\right)\right)$ so there exists $x^{\prime} \in^{*}\left(A^{n} M^{\prime}\right)$ such that ${ }^{*} g(x)={ }^{*} g\left(x^{\prime}\right)$; that is, ${ }^{*} g\left(x-x^{\prime}\right)=0$. Therefore since ${ }^{*} M \stackrel{*_{f}}{\rightarrow}\left(M^{\prime}\right)$ $\stackrel{*_{0} *}{\rightarrow}\left(M^{\prime \prime}\right)$ is exact, $x-x^{\prime} \in{ }^{*} f\left({ }^{*} M\right)$; that is $x \in x^{\prime}+{ }^{*} f\left({ }^{*} M\right) \subseteq{ }^{*} f\left({ }^{*} M\right)$ $+^{*}\left(A^{n} M^{\prime}\right)$ and $\mu^{\prime}(x) \in\left({ }^{*} f\left({ }^{*} M\right)+\mu^{\prime}\right) / \mu^{\prime}=f_{\mu}\left(M_{\mu}\right)$. It follows that $\operatorname{Ker} g_{\mu} \subseteq \operatorname{Im} f_{\mu}$. The reverse inclusion is easy.

We make $M \otimes_{R} R_{\mu}$ into an $R_{\mu}$-module by defining $\mu(a)(x \otimes \mu(b))$ $=x \otimes \mu(a b)$ for $a, b \in \in^{*} R, x \in M$. The preceding lemma can be used to prove that $M_{\mu} \cong M \otimes_{R} R_{\mu}$; the proof is completely analogous to the proof in $[12$, pp. $265-266]$ that $M^{*} \cong M \otimes_{R} R^{*}$ so it will be omitted here.

TheOREM 1. If $(R, A)$ is a Zariski ring then $\left(R, R_{\mu}\right)$ is a flat couple.

Proof. $R$ is a subring of $R_{\mu}$ and they share a common identity. If $B$ is an ideal of $R$ then it is easy to show that $B R_{\mu} \cap R=B$ since $B$ is closed in $R$. To show that $R_{\mu}$ is a flat $R$-module, suppose that $M$ and $M^{\prime}$ are $R$-modules and $f: M \rightarrow M^{\prime}$ is an injective $R$-module homomorphism. It is sufficient to prove the result when $M$ and $M^{\prime}$ are finitely generated. By the preceding lemma, $f_{\mu}: M_{\mu} \rightarrow M_{\mu^{\prime}}^{\prime}$ is an injective homomorphism. It is easily verified that

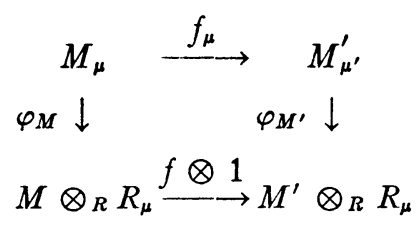

is commutative, where $\varphi_{M}, \varphi_{M}$, are isomorphisms. Hence $f \otimes 1$ is injective.

It is known that flatness implies the following [2, pp. 83-84].

Corollary. If $(R, A)$ is a Zariskiring, $B$ is an ideal of $R$, and $L$ and $N$ submodules of $M$, then

(i) $(L \cap N)_{\mu}=(L \cap N) R_{\mu}=L R_{\mu} \cap N R_{\mu}=L_{\mu} \cap N_{\mu}$,

(ii) $(N: B)_{\mu}=(N: B) R_{\mu}=N R_{\mu}: B R_{\mu}=N_{\mu}: B_{\mu}$,

(iii) $(L: N)_{\mu}=(L: N) R_{\mu}=L R_{\mu}: N R_{\mu}=L_{\mu}: N_{\mu}$.

3. A Noetherian extension. Throughout this section $A$ will denote any ideal of $R$ (not necessarily Zariski) such that $\bigcap_{n \in I} A^{n}=(0)$, and the monad of zero in ${ }^{*} R$, $\mu$, will always be computed relative to this $A$; that is, $R_{\mu}={ }^{*} R / \mu, \mu=\bigcap_{n \in I} *\left(A^{n}\right)$. $\operatorname{Rad} R$ will denote the Jacobson radical of $R$.

If $A^{n}=(0)$ for some $n \in I$ then $R_{\mu}={ }^{*} R$ and elementary properties of enlargements make it easy to show that $R_{\mu}$ is Noetherian if and only if $R$ is Artinian (also if and only if $R_{\mu}$ is Artinian). 
THEOREM 2. If $\left(R ; P_{1}, \cdots, P_{k}\right)$ is a semilocal ring and $A=\operatorname{Rad} R$, then $\left(R_{\mu} ;\left(P_{1}\right)_{\mu}, \cdots,\left(P_{k}\right)_{\mu}\right)$ is a semilocal ring.

Proof. For $1 \leqq i \leqq k, P_{i}$ a maximal ideal of $R$ means that $P_{\boldsymbol{i}}$ is an ideal and for every element $a$ of $R$ not in $P_{i}$ there is an element $b$ in $R$ such that $a b-1 \in P_{i}$. Interpreting this in the enlargement, we see that ${ }^{*} P_{\boldsymbol{i}}$ must be a maximal ideal among all ideals (internal and external) of ${ }^{*} R$. Since $A \subseteq P_{i}$ it is easy to show that $\left(P_{i}\right)_{\mu} \neq R_{\mu}$ so that the $\left(P_{i}\right)_{\mu}$ are maximal ideals of $R_{\mu}$.

If $Q$ is a maximal ideal of $R_{\mu}$ then $\mu^{-1}(Q)$ is a maximal ideal of ${ }^{*} R$. Since $P_{1}, \cdots, P_{k}$ are finitely generated the standard and nonstandard meaning of the product ${ }^{*} P_{1} \ldots{ }^{*} P_{k}$ coincide, so we can use the symbol without ambiguity. We have

$$
{ }^{*} P_{1} \ldots{ }^{*} P_{k} \subseteq{ }^{*} P_{1} \cap \cdots \cap{ }^{*} P_{k}={ }^{*}\left(P_{1} \cap \cdots \cap P_{k}\right)={ }^{*}(\operatorname{Rad} R) .
$$

But $x \in \operatorname{Rad} R$ if and only if for every $y \in R$ there is a $z \in R$ such that $(1-x y) z=1$. Interpreting this in the enlargement ${ }^{*} R$, we see that ${ }^{*}(\operatorname{Rad} R)=\operatorname{Rad}{ }^{*} R$. It follows that ${ }^{*} P_{1} \ldots{ }^{*} P_{k} \subseteq \operatorname{Rad}{ }^{*} R \subseteq \mu^{-1}(Q)$ and since $\mu^{-1}(Q)$ is prime, being maximal, ${ }^{*} P_{i} \subseteq \mu^{-1}(Q)$ for some $i=1, \cdots, k$. But ${ }^{*} P_{i}$ is a maximal ideal so ${ }^{*} P_{i}=\mu^{-1}(Q)$ and it follows that $Q=\left(P_{i}\right)_{\mu}$. Hence $\left(P_{1}\right)_{\mu}, \cdots,\left(P_{k}\right)_{\mu}$ are the maximal ideals of $R_{\mu}$ and they are finitely generated.

We now have a ring $R_{\mu}$ which Nagata calls a "semilocal ring which may not be Noetherian" [5, p. 13]. To show it is Noetherian it only remains to show that every finitely generated ideal of $R_{\mu}$ is a closed subset of $R_{\mu}$ [5, p. 110]. So let $Q$ be a finitely generated ideal of $R_{\mu}$; say its generators are $\mu\left(a_{1}\right), \cdots, \mu\left(a_{m}\right), a_{i} \in{ }^{*} R$, and let $Q^{\prime}$ ${ }^{*} R a_{1}+\cdots+{ }^{*} R a_{m}$ (note that $Q^{\prime}$ is an internal ideal of ${ }^{*} R$ ). If $\mu(x) \notin Q$ then $x \notin Q^{\prime}+\mu=\bigcap_{n \in I}\left(Q^{\prime}+^{*}\left(A^{n}\right)\right)$. There is an $n \in I$ such that $x \notin Q^{\prime}+{ }^{*}\left(A^{n}\right)$ and therefore $x+{ }^{*}\left(A^{n}\right)$ does not intersect $Q^{\prime}+{ }^{*}\left(A^{n}\right)$ or $Q^{\prime}+\mu$. It follows that $\mu(x)+\left(A^{n}\right)_{\mu}$ does not intersect $Q$ so that $Q$ is closed.

Theorem 3. If $R_{\mu}$ is Noetherian then $R_{\mu}$ is semilocal.

Proof. If $R_{\mu}$ is Noetherian then $A$ is contained in only finitely many maximal ideals of $R$. For suppose $A$ is contained in infinitely many distinct maximal ideals. Then for each $n \in I$ there is a strictly increasing sequence of ideals of length $n$ which includes $A$. Thus in the enlargement there is a sequence of (internal) ideals of length $w$, where $w$ is an infinite natural number. The $\mu$-image of these ideals will give a standard infinite strictly increasing sequence of ideals in $R_{\mu}$, contrary to $R_{\mu}$ being Noetherian. 
So suppose that $P_{1}, \cdots, P_{k}$ are the maximal ideals of $R$ containing $A .\left(P_{1}\right)_{\mu}, \cdots,\left(P_{k}\right)_{\mu}$ are maximal ideals of $R_{\mu}$. Suppose $Q$ is a maximal ideal of $R_{\mu}$; let $\mu\left(a_{1}\right), \cdots, \mu\left(a_{m}\right)$ be its generators and set $Q^{\prime}={ }^{*} R a_{1}+\cdots+{ }^{*} R a_{m}$. Since $Q$ is maximal, $Q^{\prime}+\mu=\mu^{-1}(Q)$ is a maximal ideal among all ideals of ${ }^{*} R$. But $Q^{\prime}+\mu \subseteq Q^{\prime}+{ }^{*} A$ so either ${ }^{*} A \subseteq Q^{\prime}+\mu$ or $Q^{\prime}+{ }^{*} A={ }^{*} R$. But this latter alternative implies (since $Q^{\prime}$ and ${ }^{*} A$ are comaximal) that for every finite $n, Q^{\prime}+\left({ }^{*} A\right)^{n}={ }^{*} R$. Hence ${ }^{*} R=\bigcap_{n \in I}\left(Q^{\prime}+{ }^{*}\left(A^{n}\right)\right)=Q^{\prime}+\mu$, a contradiction. Therefore ${ }^{*} A \subseteq Q^{\prime}+\mu=\bigcap_{n \in I}\left(Q^{\prime}+{ }^{*}\left(A^{n}\right)\right)$. Since this last intersection is not ${ }^{*} R$, there is a finite $n \in I$ such that ${ }^{*} A \subseteq Q^{\prime}+^{*}\left(A^{n}\right) \neq{ }^{*} R$. Now in $R$ it is true that for any $m$ elements $b_{1}, \cdots, b_{m}$ of $R$, if $A \subseteq R b_{1}+\cdots+R b_{m}$ $+A^{n} \neq R$ then each of the $b_{i}(1 \leqq i \leqq m)$ belong to $P_{j}$ for some $j$, $1 \leqq j \leqq k$, (since $R b_{1}+\cdots+R b_{k}+A^{n} \subseteq P_{j}$ for some $j$ ). Interpreting this sentence in ${ }^{*} R$, we have each of the $a_{i}$ in ${ }^{*} P_{j}$ for some $j, 1 \leqq j \leqq k$. It follows that $Q^{\prime}+\mu={ }^{*} P_{j}+\mu$ and $Q=\left(P_{i}\right)_{\mu}$. Thus $R_{\mu}$ is a semilocal ring.

The preceding theorems and their proofs imply the following.

THEOREM 4. The following are equivalent for a Noetherian ring $R$ with $A=\operatorname{Rad} R$.

(i) $R$ is semilocal.

(ii) $R_{\mu}$ is semilocal.

(iii) $\left(R_{\mu}, A R_{\mu}\right)$ is a Zariski ring.

(iv) $R_{\mu}$ is Noetherian.

Remarks. Let $\left(R ; P_{1}, \cdots, P_{k}\right)$ be a semilocal ring and $A=\operatorname{Rad} R$. Since every ideal of $R_{\mu}$ is finitely generated, every ideal of $R_{\mu}$ is the homomorphic image of an internal ideal of $* R$. Thus any sentence about $R$ and its ideals whose truth is preserved under homomorphic images will remain true for $R_{\mu}$ and its ideals. But Theorems 1 and 2 are sufficient to say even more about the ideal structures of $R$ and $R_{\mu}$; for example, even though the lattice of ideals of $R_{\mu}$ (strictly) contains the lattice of ideals of $R$, we can still conclude from these results that the (Krull) dimensions of $R$ and $R_{\mu}$ are equal. In fact, all the results contained in [10] (which includes the above claim) continue to hold with $\hat{R}$ (the completion of $R$ ) replaced by $R_{\mu}$. All the proofs in that paper, with the exception of Proposition 2 are identical. Proposition 2 can be proved exactly as Lemma 2 to Theorem 30 in [12, p. 312].

Example. Let $R$ be a semilocal ring and $A=\operatorname{Rad} R$. Define $R_{1}=R$, $A_{1}=A, \mu_{1}=\mu$ and for $m>1$ define $R_{m}=\left(R_{m-1}\right)_{\mu_{m-1}}, A_{m}=\operatorname{Rad} R_{m}$ $=\left(\operatorname{Rad} R_{m-1}\right)_{\mu_{m-1}}$, and $\mu_{m}=\bigcap_{n \in I} *\left(A_{m}^{n}\right)$. If $R / A^{k}$ is infinite for some $k$ then we have a strictly increasing (the canonical mapping $R / A^{k}$ 
$\rightarrow R_{\mu} /\left(A^{k}\right)_{\mu}$ is one-to-one; it is onto if and only if $R / A^{k}$ is finite) sequence of semilocal rings, each of the same dimension, each with the same number of maximal ideals, and $\left(R_{n}, R_{m}\right)$ is a flat couple for all $n, m \in I, 1 \leqq n \leqq m$.

\section{BIBLIOGRAPHY}

1. N. Bourbaki, Algèbre commutative. Chapitres 1, 2, 3, Actualités Sci. Indust., nos. 1290, 1293, Hermann, Paris, 1961. MR 30 \#2027; MR $36 \# 146$.

2. Christer Lech, Note on multiplicities of ideals, Ark. Mat. 4 (1960), 63-86. MR 25 \#3955.

3. W. A. J. Luxemburg (Editor), Applications of model theory to algebra, analysis, and probability, Holt, Rinehart and Winston, New York, 1969. MR 38 \#3143.

4. M. Machover and J. Hirschfeld, Lectures on non-standard analysis, Lecture Notes in Math., no. 94, Springer-Verlag, Berlin and New York, 1969. MR 40 \#2531.

5. Masayoshi Nagata, Local rings, Interscience Tracts in Pure and Appl. Math., no. 13, Interscience, New York, 1962. MR 27 \#5790.

6. Abraham Robinson, Non-standard analysis, Studies in Logic and the Foundations of Mathematics, North-Holland, Amsterdam, 1966. MR 34 \#5680.

7. - Non-standard arithmetic, Bull. Amer. Math. Soc. 73 (1967), 818-843. MR $36 \# 1319$.

8. - Non-standard theory of Dedekind rings, Nederl. Akad. Wetensch. Proc. Ser. A $70=$ Indag. Math. 29 (1967), 444-452. MR 36 \#6399.

9. - Compactification of groups and rings and non-standard analysis, J. Symbolic Logic 34 (1969), 576-588.

10. Hazimu Satô, Some remarks on Zariski rings, J. Sci. Hiroshima Univ. Ser. A 20 (1956/57), 93-99. MR 20 \#2335.

11. O. Zariski and P. Samuel, Commutative algebra. Vol. 1, University Series in Higher Math., Van Nostrand, Princeton, N. J., 1958. MR 19, 833.

12. - Commutative algebra. Vol. 2, University Series in Higher Math., Van Nostrand, Princeton, N. J., 1960. MR 22 \#11006.

St. Olaf College, Northfield, Minnesota 55057 